\title{
The ponderomotive force of an electromagnetic wave in a collisional plasma
}

\author{
By M. L. SAWLEY \\ Centre de Recherches en Physique des Plasmas, Association Euratom, \\ Confédération Suisse, Ecole Polytechnique Fédérale de Lausanne, \\ 21, av. des Bains, CH-1007 Lausanne/Switzerland
}

(Received 3 September 1984)

The nonlinear propagation of a circularly polarized, electromagnetic wave in a collisional, infinite, magnetized plasma is considered. The presence of collisions leads to spatial variation in the amplitude of the wave field which gives rise to a time-independent ponderomotive force. The ponderomotive potential for a left (right) circularly polarized wave attains a maximum at the ion (electron) cyclotron frequency. In the vicinity of the cyclotron frequency it is shown to be always positive. A decrease in both the particle density and the real and imaginary parts of the complex wavenumber is shown to result from the effect of the ponderomotive force.

\section{Introduction}

It is well known that spatial gradients in the amplitude of an oscillating electric field can give rise to a time-independent, ponderomotive force. This force plays a fundamental role in nonlinear plasma dynamics owing to its influence on the equilibrium plasma parameters. Considerable effort has been made in the past to obtain a general expression for the ponderomotive force in a collisionless magnetized plasma. (An extensive review of this work is given by Statham \& ter Haar (1983).)

In a bounded plasma, electric field gradients may be externally imposed by a localized antenna. Numerous authors have considered the effect of the ponderomotive force for such a non-self-consistent electromagnetic field on, for example, the acceleration of charged particles (Consoli \& Hall 1963), and the radiofrequency plugging (Eubank 1969; Fader et al. 1981) and low-frequency mode stabilization (Ferron et al. 1983; Yasaka \& Itatani 1984) of open-ended mirror devices.

For a collisionless, infinite, magnetized plasma, Festeau-Barrioz \& Weibel (1980) have investigated the ponderomotive force due to an ion cyclotron wave which, for spatial gradients in the amplitude of the self-consistent electric field to exist, must be either a standing wave or a wave beyond cut-off. An expression for the ponderomotive force, exerted by a circularly polarized wave with gradients in the direction of the steady magnetic field, was obtained in terms of the gradient of a quasi-potential. This ponderomotive potential is zero, in the laboratory frame, for an undamped wave propagating in an infinite plasma 
(although, as discussed by Roberts \& Buchsbaum (1964), a travelling wave can create a quasi-potential in the wave frame).

In the present paper, we consider a self-consistent treatment of the propagation of a circularly polarized, electromagnetic wave along the direction of a steady magnetic field in a collisional, infinite plasma. The presence of collisions leads to a spatial decay of the electric field amplitude, the decay being exponential for a wave of sufficiently small amplitude. We show that this spatial variation of the wave field gives rise to a non-zero, time-independent ponderomotive force. Solving the nonlinear equations to second order in electric field strength, an expression for the ponderomotive potential is obtained. The modification of the particle densities and the complex wavenumber (that is, the phase velocity and attenuation length) that results from the influence of the ponderomotive force is calculated.

\section{Basic equations}

The nonlinear propagation of an electromagnetic wave in a collisional, warm plasma may be adequately described by the self-consistent solution of the following set of multi-fluid equations: the equation of motion for species $\sigma$,

$$
n_{\sigma} m_{\sigma}\left(\frac{\partial \mathbf{u}_{\sigma}}{\partial t}+\mathbf{u}_{\sigma} . \nabla \mathbf{u}_{\sigma}\right)=n_{\sigma} q_{\sigma}\left(\mathbf{E}+\mathbf{u}_{\sigma} \times \mathbf{B}\right)-\nabla p_{\sigma}-n_{\sigma} m_{\sigma} \nu_{\sigma} \mathbf{u}_{\sigma}
$$

the equation of continuity,

Maxwell's equations,

$$
\partial n_{\sigma} / \partial t+\nabla \cdot\left(n_{\sigma} \mathbf{u}_{\sigma}\right)=0
$$

and the equation of state,

$$
\begin{gathered}
\nabla \times \mathbf{E}=-\partial \mathbf{B} / \partial t \\
\nabla \times \mathbf{B}=\mu_{0} \sum_{\sigma} n_{\sigma} q_{\sigma} \mathbf{u}_{\sigma}+\frac{1}{c^{2}} \frac{\partial \mathbf{E}}{\partial t}
\end{gathered}
$$

$$
p_{\sigma}=n_{\sigma} T_{\sigma}
$$

In (1), a simple form for the collision term, namely $-n_{\sigma} m_{\sigma} \nu_{\sigma} \mathbf{u}_{\sigma}$, has been used to avoid unnecessary mathematical complications. This collision term is appropriate, for example, to describe charged particle-neutral collisions in a weakly ionized plasma. We shall assume that the temperature $T_{\sigma}$ of each species is constant, and unaffected by the presence of the wave.

The equilibrium quantities, in the absence of the wave, are

$$
\mathbf{B}=B_{0} \hat{\mathbf{z}}, \quad n_{\sigma}=\bar{n}_{\sigma}
$$

where $B_{0}$ and $\bar{n}_{\sigma}$ are constants in space and time. The equilibrium values of $\mathbf{E}$ and $\mathbf{u}_{\sigma}$ are assumed to be zero.

We shall consider the propagation in the positive $\hat{\mathbf{z}}$ direction (i.e. parallel to $B_{0}$ ) of a circularly polarized electromagnetic wave with an electric wave field of the form

where

$$
\mathbf{E}_{\omega}(z, t)=E_{0}(1, \pm i, 0) \exp [i(K z-\omega t)]
$$

$$
K=k(z)+i \gamma(z), \quad(k, \gamma \text { real })
$$


and $E_{0}$, the amplitude at $z=0$, is constant in time. In (7), the upper (lower) sign refers to the right (left) circularly polarized wave.

Using (3) and (7), the magnetic field component of the wave may be obtained:

$$
\mathbf{B}_{\omega}(z, t)=\frac{1}{\omega}\left(K+\frac{d K}{d z} z\right) E_{0}(\mp i, 1,0) \exp [i(K z-\omega t)] .
$$

Equation (1) can then be split into the perpendicular and parallel components:

$$
\begin{gathered}
n_{\sigma} m_{\sigma} \frac{\partial \mathbf{u}_{\sigma}}{\partial t}=n_{\sigma} q_{\sigma}\left(\mathbf{E}_{\omega}+\mathbf{u}_{\sigma} \times \mathbf{B}_{0}\right)-n_{\sigma} m_{\sigma} \nu_{\sigma} \mathbf{u}_{\sigma} \\
n_{\sigma} q_{\sigma}\left(E_{z}+\mathbf{u}_{\sigma} \times \mathbf{B}_{\omega}\right)-\frac{\partial p_{\sigma}}{\partial z}=0 .
\end{gathered}
$$

Equation (10) is linear in the wave quantities. It yields the following fluid velocities for species $\sigma$ :

$$
\mathbf{u}_{\sigma}(z, t)=\frac{q_{\sigma} E_{0}}{m_{\sigma}\left(\omega \pm \Omega_{\sigma}+i \nu_{\sigma}\right)}(i, \mp 1,0) \exp [i(K z-\omega t)]
$$

where $\Omega_{\sigma}=q_{\sigma} B_{0} / m_{\sigma}$ is the cyclotron frequency for species $\sigma$.

The force balance along the direction of the magnetic field $\mathbf{B}_{0}$, is given by (11). Taking the real part of (9) and (12), it may be shown that for a circularly polarized wave propagating in a collisional plasma, the nonlinear (ponderomotive) force exerted by the wave on each species is independent of time and given by

$$
q_{\sigma}\left(\mathbf{u}_{\sigma} \times \mathbf{B}_{\omega}\right)=\hat{\mathbf{z}} \frac{q_{\sigma}^{2} E_{0}^{2} e^{-2 \gamma z}}{m_{\sigma} \omega\left[\left(\omega \pm \Omega_{\sigma}\right)^{2}+\nu_{\sigma}^{2}\right]} \frac{d}{d z}\left[\nu_{\sigma} k z+\left(\omega \pm \Omega_{\sigma}\right) \gamma z\right] .
$$

In the steady state, the ponderomotive force is balanced by axial pressure gradients and the time-independent electrostatic field $E_{z}$, which arise from the spatial separation of the different species.

The electric wave field given by (7) must satisfy the wave equation obtained from (3) and (4):

$$
\frac{\partial^{2} \mathbf{E}_{\omega}}{\partial z^{2}}=\mu_{0} \sum_{\sigma} n_{\sigma} q_{\sigma} \frac{\partial \mathbf{u}_{\sigma}}{\partial t}+\frac{1}{c^{2}} \frac{\partial^{2} \mathbf{E}_{\omega}}{\partial t^{2}} .
$$

Substituting from (7) and (12) yields the nonlinear dispersion relation

$$
\left(\frac{d K z}{d z}\right)^{2}-i \frac{d^{2} K z}{d z^{2}}-\frac{\omega^{2}}{c^{2}}\left[1-\sum_{\sigma} \frac{\omega_{p \sigma}^{2}}{\omega\left(\omega \pm \Omega_{\sigma}+i \nu_{\sigma}\right)}\right]=0,
$$

where $\omega_{p \sigma}=\left(n_{\sigma} q_{\sigma}^{2} / m_{\sigma} \epsilon_{0}\right)^{\frac{1}{2}}$ is the plasma frequency for species $\sigma$.

\section{The linear wavenumber}

If we assume that the wave amplitude is sufficiently small to neglect the nonlinear interaction, the wavenumber (denoted as $K_{0}=k_{0}+i \gamma_{0}$ for the linear solution) is independent of $z$ and given, using (14), by

$$
K_{0}^{2}-\frac{\omega^{2}}{c^{2}}\left[1-\sum_{\sigma} \frac{\overline{\omega_{p \sigma}^{2}}}{\omega\left(\omega \pm \Omega_{\sigma}+i \nu_{\sigma}\right)}\right]=0
$$


This is the usual form of the linear dispersion relation for an electromagnetic wave propagating in a collisional plasma (Ginzburg, 1961).

Defining

and

$$
\left.\begin{array}{c}
A=1-\sum_{\sigma} \frac{\overline{\omega_{p \sigma}^{2}}\left(\omega \pm \Omega_{\sigma}\right)}{\omega\left[\left(\omega \pm \Omega_{\sigma}\right)^{2}+\nu_{\sigma}^{2}\right]}, \\
B=\sum_{\sigma} \frac{\overline{\omega_{p \sigma}^{2}} \nu_{\sigma}}{\omega\left[\left(\omega \pm \Omega_{\sigma}\right)^{2}+\nu_{\sigma}^{2}\right]},
\end{array}\right\}
$$

the real and imaginary parts of the complex wavenumber may be expressed as

$$
\left.\begin{array}{l}
k_{0}^{2}=\frac{1}{2} \frac{\omega^{2}}{c^{2}}\left[A+\left(A^{2}+B^{2}\right)^{\frac{1}{2}}\right], \\
\gamma_{0}^{2}=\frac{1}{2} \frac{\omega^{2}}{c^{2}}\left[-A+\left(A^{2}+B^{2}\right)^{\frac{1}{2}}\right] .
\end{array}\right\}
$$

In the presence of collisions, the wave does not suffer from the effects of resonance and cut-off; for all frequencies the wave possesses a non-zero, finite value of $k_{0}$. However, in the frequency ranges of cut-off for the undamped wave, we find $\gamma_{0} \gg k_{0}$, that is, the wave is heavily damped.

For future reference, we note that the ratio of real and imaginary parts of the complex wavenumber may be written as

$$
\frac{k_{0}}{\gamma_{0}}=\frac{A}{B}+\left(1+\frac{A^{2}}{B^{2}}\right)^{\frac{1}{2}}
$$

If the collision frequency $\nu_{\sigma}$ is sufficiently small, then for $\omega \simeq \pm \Omega_{\sigma}$ we may approximate $A$ and $B$ by the contribution due to the species $\sigma$ alone. Thus

$$
A \simeq-\frac{\overline{\omega_{p \sigma}^{2}}\left(\omega \pm \Omega_{\sigma}\right)}{\omega\left[\left(\omega \pm \Omega_{\sigma}\right)^{2}+\nu_{\sigma}^{2}\right]}, \quad B \simeq \frac{\overline{\omega_{p \sigma}^{2}} \nu_{\sigma}}{\omega\left[\left(\omega \pm \Omega_{\sigma}\right)^{2}+\nu_{\sigma}^{2}\right]},
$$

and, substituting into (18),

$$
\frac{\nu_{\sigma} k_{0}}{\gamma_{0}} \simeq-\left(\omega \pm \Omega_{\sigma}\right)+\left[\left(\omega \pm \Omega_{\sigma}\right)^{2}+\nu_{\sigma}^{2}\right]^{\frac{1}{2}}
$$

\section{The nonlinear solution}

The axial force balance equation (11) may be solved to second order in electric field strength by substituting the wavenumber to first order, calculated in $\S 3$, into the expression (13) for the ponderomotive force. We then obtain

$$
q_{\sigma}\left(\mathbf{u}_{\sigma} \times \mathbf{B}_{\omega}\right)=-\frac{d \Phi_{\sigma}}{d z} \hat{\mathbf{z}}
$$

where the ponderomotive potential for species $\sigma$ is given, for $\gamma_{0} \neq 0$, by

$$
\Phi_{\sigma}=\frac{q_{\sigma}^{2} E_{0}^{2} e^{-2 \gamma_{0} z}}{2 m_{\sigma}\left[\left(\omega \pm \Omega_{\sigma}\right)^{2}+\nu_{\sigma}^{2}\right]}\left[\left(\omega \pm \Omega_{\sigma}\right)+\frac{\nu_{\sigma} k_{0}}{\gamma_{0}}\right]
$$


Writing the axial electrostatic field as the gradient of a scalar potential,

we obtain from (11), using (5),

$$
E_{z}=-d U / d z
$$

$$
-q_{\sigma} \frac{d U}{d z}-\frac{d \Phi_{\sigma}}{d z}-\frac{T_{\sigma}}{n_{\sigma}} \frac{d n_{\sigma}}{d z}=0 .
$$

The solution to second order in electric field strength is

$$
n_{\sigma}(z)=\bar{n}_{\sigma}\left[1-\frac{\left(q_{\sigma} U+\Phi_{\sigma}\right)}{T_{\sigma}}\right]
$$

where we have used the boundary condition, $n_{\sigma}(z \rightarrow \infty)=\bar{n}_{\sigma}$.

The electrostatic potential $U$ may be obtained by imposing the condition that the plasma is quasi-neutral, that is,

Substituting from (21) yields

$$
\sum_{\sigma} n_{\sigma} q_{\sigma}=0
$$

Defining

$$
U=-\frac{\sum_{\sigma} \bar{n}_{\sigma} q_{\sigma} \Phi_{\sigma} / T_{\sigma}}{\sum_{\sigma} \bar{n}_{\sigma} q_{\sigma}^{2} / T_{\sigma}}
$$

and

$$
\epsilon_{\sigma}=\frac{q_{\sigma}^{2}\left[\left(\omega \pm \Omega_{\sigma}\right)+\nu_{\sigma} k_{0} / \gamma_{0}\right]}{2 m_{\sigma} \omega\left[\left(\omega \pm \Omega_{\sigma}\right)^{2}+\nu_{\sigma}^{2}\right]}
$$

$$
\mathscr{U}=-\frac{\sum_{\sigma} \bar{n}_{\sigma} q_{\sigma} \epsilon_{\sigma} / T_{\sigma}}{\sum_{\sigma} \bar{n}_{\sigma} q_{\sigma}^{2} / T_{\sigma}}
$$

the density of species $\sigma$, to second order in electric field strength, may be written as

$$
n_{\sigma}(z)=\bar{n}_{\sigma}\left[1-\frac{\left(q_{\sigma} \mathscr{U}+\epsilon_{\sigma}\right)}{T_{\sigma}} E_{0}^{2} e^{-2 \gamma_{0} z}\right]
$$

The electric field therefore produces a nonlinear modification of the density of each particle species. The change in density causes, in turn, a change in the wavenumber. Substituting (24) into (14), the modified wavenumber satisfies the following nonlinear dispersion relation:

where

$$
\left(\frac{d K z}{d z}\right)^{2}-i \frac{d^{2} K z}{d z^{2}}-K_{0}^{2}\left(1+\alpha E_{0}^{2} e^{-2 \gamma_{0} z}\right)=0,
$$

$$
\alpha=\frac{\omega^{2}}{K_{0}^{2} c^{2}} \sum_{\sigma} \frac{\overline{\omega_{p \sigma}^{2}}}{\omega\left(\omega \pm \Omega_{\sigma}+i \nu_{\sigma}\right)} \frac{\left(q_{\sigma} \mathscr{U}+\epsilon_{\sigma}\right)}{T_{\sigma}} .
$$

The solution of (25) that satisfies the boundary conditions

$$
K(z=0) \text { finite, } K(z \rightarrow \infty)=K_{0},
$$

is, to second order in electric field strength,

$$
K(z)=K_{0}+\frac{\alpha K_{0}^{2} E_{0}^{2}}{4\left(K_{0}+i \gamma_{0}\right)} \frac{\left(1-e^{-2 \gamma_{0} z}\right)}{\gamma_{0} z} .
$$




\section{Ponderomotive effect for $\omega \simeq \pm \Omega_{\sigma}$}

It was shown in $\S 4$ that the nonlinear force exerted by an electromagnetic wave in a collisional plasma may be expressed, for each particle species $\sigma$, as the gradient of a ponderomotive potential, $\Phi_{\sigma}$. This potential, in addition to exhibiting the usual dependence on $\left|E_{\omega}\right|^{2}$, has also, in general, a dependence on the wave properties through the parameter $\nu_{\sigma} k_{0} / \gamma_{0}$. However, if $\nu_{\sigma}$ is sufficiently small, then for $\omega \simeq \pm \Omega_{\sigma}$ we may substitute into (20) the approximate value of this parameter given by (19). We then obtain

where

$$
\Phi_{\sigma} \simeq \frac{q_{\sigma}^{2} E_{0}^{2} e^{-2 \gamma_{0} z}}{2 m_{\sigma} \Omega_{\sigma}^{2}} \Psi_{\sigma}^{ \pm}\left(\frac{\omega}{\Omega_{\sigma}}, \frac{\nu_{\sigma}}{\Omega_{\sigma}}\right)
$$

$$
\Psi_{\sigma}^{ \pm}\left(\frac{\omega}{\Omega_{\sigma}}, \frac{\nu_{\sigma}}{\Omega_{\sigma}}\right)=\left\{\frac{\omega}{\Omega_{\sigma}}\left[\left(1 \pm \frac{\omega}{\Omega_{\sigma}}\right)^{2}+\frac{\nu_{\sigma}^{2}}{\Omega_{\sigma}^{2}}\right]^{\frac{1}{2}}\right\}^{-1} .
$$

The ponderomotive potential for each particle species, in the presence of a wave with appropriate polarization, attains a maximum at the cyclotron frequency of that species. In addition, (28) shows that it is always positive in the vicinity of the cyclotron frequency. We note that this is contrary to the case of an electromagnetic wave in a collisionless plasma. The standard expression for the ponderomotive potential (Motz \& Watson 1967),

$$
\Phi_{\sigma}=\frac{q_{\sigma}^{2}|E(z)|^{2}}{2 m_{\sigma} \omega\left(\omega \pm \Omega_{\sigma}\right)},
$$

reveals a change of sign of the left (right) circularly polarized wave as the frequency crosses the ion (electron) cyclotron frequency. However, care must be taken when applying expression (29) if the electric field $E(z)$ is calculated selfconsistently. For a collisionless plasma, the wave is propagatory for $\omega \lesssim\left|\Omega_{\sigma}\right|$ but evanescent for $\omega \gtrsim\left|\Omega_{\sigma}\right|$. Therefore the wave must satisfy different boundary conditions in the different frequency regimes (Festeau-Barrioz \& Sawley 1984). Hence, expression (29) cannot be applied to a self-consistent wave in a continuous fashion as the wave frequency crosses the cyclotron frequency.

If we consider a plasma for which $\left|q_{\sigma}\right|$ and $T_{\sigma}$ are the same for all particle species, then for $\omega \simeq \pm \Omega_{\sigma}$ we may approximate (23) as

Therefore

$$
\mathscr{U} \simeq-\frac{\bar{n}_{\sigma} \epsilon_{\sigma}}{2 \bar{n}_{e} q_{\sigma}}
$$

$$
\frac{q_{\sigma} \mathscr{U}+\epsilon_{\sigma}}{T_{\sigma}} \simeq \frac{q_{\sigma}^{2}}{2 m_{\sigma} \Omega_{\sigma}^{2} T_{\sigma}}\left(1-\frac{\bar{n}_{\sigma}}{2 \bar{n}_{e}}\right) \Psi_{\sigma}^{ \pm}>0,
$$

and from (24) it may be seen that the ponderomotive force causes a decrease in the particle density of species $\sigma$.

Writing

$$
k=k_{0}(1+\Delta k), \quad \gamma=\gamma_{0}(1+\Delta \gamma)
$$


then from (27) we may obtain approximate expressions for the modification of the real and imaginary parts of the complex wavenumber for $\omega \simeq \pm \Omega_{\sigma}$ :

where

$$
\begin{aligned}
& \Delta k \simeq-\beta\left\{\left(\omega \pm \Omega_{\sigma}\right)+2\left[\left(\omega \pm \Omega_{\sigma}\right)^{2}+\nu_{\sigma}^{2}\right]^{\frac{1}{2}}\right\} E_{0}^{2} \frac{\left(1-e^{-2 \gamma_{0} z}\right)}{\gamma_{0} z}, \\
& \Delta \gamma \simeq-\beta\left\{\left(\omega \pm \Omega_{\sigma}\right)+\left[\left(\omega \pm \Omega_{\sigma}\right)^{2}+\nu_{\sigma}^{2}\right]^{\frac{1}{2}}\right\} E_{0}^{2} \frac{\left(1-e^{-2 \gamma_{0} z}\right)}{\gamma_{0} z},
\end{aligned}
$$

$$
\beta=\frac{\overline{\omega \omega_{p \sigma}^{2}}}{4\left(k_{0}^{2}+4 \gamma_{0}^{2}\right) c^{2}} \frac{1}{\left[\left(\omega \pm \Omega_{\sigma}\right)^{2}+\nu_{\sigma}^{2}\right]} \frac{\left(q_{\sigma} \mathscr{U}+\epsilon_{\sigma}\right)}{T_{\sigma}} .
$$

From (30), $\beta>0$, and therefore the decrease in particle density that results from the effect of the ponderomotive force for $\omega \simeq \pm \Omega_{\sigma}$, causes a decrease in both the real and imaginary parts of the complex wavenumber. Thus, as a result of the ponderomotive force, both the phase velocity $(\omega / k)$ and the attenuation length $(1 / \gamma)$ are increased. The nonlinear density modification caused by the ponderomotive force therefore acts to oppose its source, that is, the spatial attenuation of the wave.

\section{Conclusions}

The spatial decay of a circularly polarized, electromagnetic wave in a collisional plasma has been shown to give rise to a time-independent, ponderomotive force. An expansion to second order in the electric field strength has been used to write this force in terms of the gradient of a ponderomotive potential. This potential attains a maximum at the cyclotron frequency of the resonant particle species. In the vicinity of the cyclotron frequency, the ponderomotive potential is always positive, and causes a decrease in the particle density which results in an increase in the phase velocity and attenuation length of the wave.

\section{REFERENCES}

Consoli, T. \& Hall, R. B. 1963 Nucl. Fusion, 3, 237.

Eubank, H. P. 1969 Phys. Fluids, 12, 234.

Fader, W. J., Jong, R. A., Stufflebeam, J. H. \& Sziklas, E. A. 1981 Phys. Rev. Lett. 46, 999.

Ferron, J. R., Hershkowitz, N., Breun, R. A., Golovato, S. N. \& Godlding, R. 1983 Phys. Rev. Lett. 51, 1955.

Festeau-Barrioz, M. C. \& Sawlex, M. L. 1984 Lausanne Report LRP 245/84.

Festeau-Barrioz, M. C. \& Weibex, E. S. 1980 Phys. Fluids, 23, 2045.

Ginzbura, V. L. 1961 Propagation of Electromagnetic Waves in Plasma. Gordon and Breach.

Motz, H. \& Watson, C. J. H. 1967 Adv. Electron. Electron Phys. 23, 153.

Roberts, C. S. \& Buchsbaum, S. J. 1964 Phys. Rev. A 135, 381.

Statham, G. \& Ter HaAr, D. 1983 Plasma Phys. 25, 681.

YaSaka, Y. \& Itatani, R. 1984 Nucl. Fusion, 24, 445. 